\title{
Application of vibration stabilizing treatment to improve the quality of aircraft parts
}

\author{
M. A. Belozerov*, V. A. Lebedev, N. S. Koval, V. V. Ivanov, and S. I. Popov \\ Don State Technical University, Rostov-on-Don, 344000, Russia
}

\begin{abstract}
The article shows that one of the most significant factors influencing a decrease in the accuracy of parts achieved during machining is the occurrence of technological residual stresses, leads to significant changes in their geometric shape. The paper reveals technological aspects of vibration stabilizing processing of parts, presents methods of its implementation, and considers possible methods of its implementation and its influence on the change in residual stresses of parts of low rigidity. The ways of increasing the efficiency of vibration stabilizing treatment ensuring its application to stabilize the quality of parts and, as a consequence, the service life of aircraft.
\end{abstract}

\section{Introduction}

Improving the reliability and durability of aircraft products is the basis for increasing the competitiveness of these products both within the Russian market and abroad. The design of such types of products includes groups of highly loaded parts that have a complex shape, large dimensions, limited rigidity and high requirements for the parameters of the surface quality and subsurface layer. As examples of such parts, one can imagine the following: a spar of a helicopter main rotor blade, elements of an aircraft wing and fuselage (panels), parts of turbojet engines and power plants, and others (Fig. 1). Many factors affect the decrease in the accuracy of these parts achieved during machining. One of the most significant factors is the occurrence of technological residual stresses, which lead to significant changes in their geometric shape $[1,2]$. To eliminate this factor, thermal, deformation and combined technological methods are used to stabilize their volumetric stress state [3, 4].

\footnotetext{
*Corresponding author: m-belozjorov@rambler.ru
} 

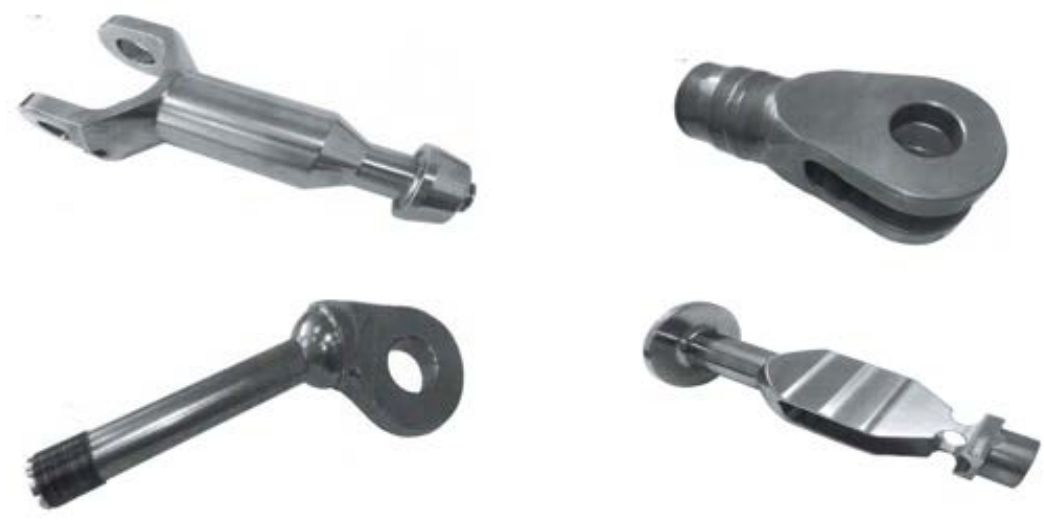

Fig. 1. Examples of power parts for aircraft

The results of theoretical and experimental studies carried out in this direction $[5,6]$ make it possible to single out vibration stabilizing treatment (VST) as a promising method of stabilizing treatment of aircraft parts, which has a number of advantages over other technological methods, the disclosure of which is the purpose of this article.

\section{Theory}

Figure 2 shows the VST technological system developed at the Vibrotechnology Research Institute of DSTU to stabilize the geometric parameters of parts, the main elements of which are: part 1, subject to stabilization; a source of periodic shock excitation of acoustic vibrations 2 ; control unit 4; supports 3 . Functionally, the stabilization process in the technological system can be implemented either by installing the part on a vibrating platform with fastening at one (two) points or by attaching the vibration source to the part mounted on a cantilever or by impact-impulse action on the part installed on elastic supports, while causing its fluctuation.

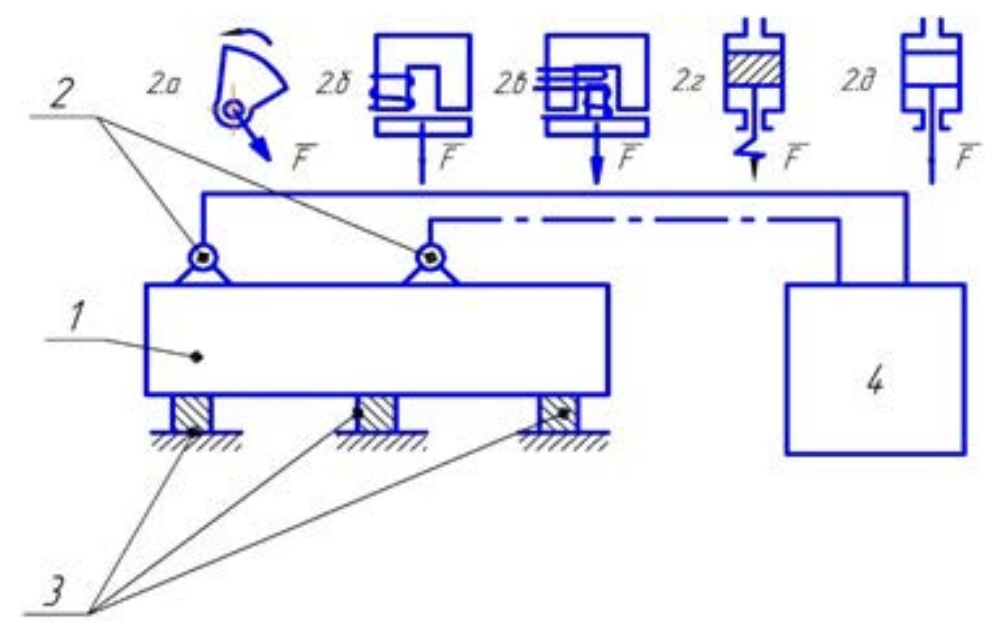

Fig. 2. VST technological system: 1-detail, 2-source of vibration (a-unbalance, b-electromagnetic, celectrodynamics, d-hydraulic, d-pneumatic), 3-supports, 4-control unit. 
The control parameters that determine the technological effect of VST are the frequency and amplitude of the vibration-exciting force, the nature and direction of its distribution, the phase shift of the vibration sources, and the location of the reference points of the part when installed on the supports. The presented technological scheme, as shown by the studies [7], allows the creation of significant cyclic loads in the entire body of the work piece due to the use of the resonant properties of its structure. It was found that VST at resonance frequencies does not give negative effects and does not affect properties such as fatigue strength indicators, and does not cause cracking phenomena. Under conditions of processing with non-resonant frequencies, due to the fact that the amplitude of the acting stresses generated by the vibration source is small, the rate of the relaxation process is low. This leads to a significant increase in the processing time and, as a rule, to the unpredictability of the stress reduction effect.

Considering the mechanism of the stabilizing effect of vibration on the material, most researchers are inclined to believe that VST process, like others belonging to the deformation class, reduces the level of residual stresses (RS) and increases the relaxation resistance of the structure due to its strengthening during micro plastic deformation of vibration creep. Analysis of the process research on cast parts made of gray iron [8], on parts (including welded ones) from low-carbon steels, parts from aluminum alloys, hot-rolled and cold-drawn low-carbon steels showed that VST of relatively non-rigid parts with high deformation capacity (welded structures, long shafts, extended castings, etc.) was generally successful in preventing warpage. This fact can be explained by the fact that the natural frequencies of the bending vibrations of the named parts were in the operating range of the applied vibration exciters. Almost all known techniques [9-11] assume the possibility of excitation at VST of at least the first natural frequency of the part. Without considering in detail the content of these techniques, it should be noted that they are applicable to VST of relatively non-rigid parts. Meanwhile, there are a large number of parts, including those in helicopter construction that have natural frequencies that exceed the limiting frequencies of unbalanced vibration exciters.

Works $[12,13]$ shows that the use of vibration stabilizing processing in the manufacture of non-rigid parts of various configurations made it possible to achieve indicators in terms of geometric accuracy that could not be achieved using other methods of stabilizing processing. In addition, to a significant increase in the geometric accuracy of parts, a decrease in residual stresses was also noted. The result of the work was applied in the production of friction discs for multi-disc oil cooled brakes at the BelAZ plant.

\section{Problems and ways to ensure dimensional stability of parts}

The part as the main element of the VST process system is an imperfectly elastic system with distributed parameters in a non-stationary complex stress state, characterized by constructive anisotropy and structural heterogeneity. Certain difficulties arise when analyzing the quality of the VST process, which are aggravated by the fact that direct control of its result dimensional stability, can be carried out only after the part has reached the limit state (violation of the accuracy standards). The issues of synthesis of control actions of the VST process, which make it possible to create vibration fields of the required amplitude and configuration in the work piece (including those with given local characteristics in the zones of concentration of operational and residual stresses), require further study in terms of justifying the choice of the fixing scheme of the part, number and location vibration sources on it, taking into account the geometry of a particular part and its vibrational properties. In this regard, for the effective implementation of vibration stabilizing treatment, a design and technological classifier of parts and recommendations for the use of various loading schemes 
should be developed, which will allow designing the most rational designs of dynamic stabilization units.

\section{Conclusions}

Analysis of works devoted to the study and application of vibration stabilizing treatment indicates the prospects of its use in the technology of manufacturing machine parts in order to ensure their quality and improve performance. Despite the fact that most of the research in this direction was carried out back in the 70-80s, they have not lost their relevance today. The method of vibration stabilizing treatment has already proved its high efficiency at various enterprises in the manufacture of friction discs, torsion and crankshafts, as well as other lowrigidity parts. However, due to insufficient elaboration, it did not receive proper application at enterprises for the manufacture of aircraft parts characterized by low structural rigidity and a tendency to form residual stresses, despite the fact that, as the analysis showed, there are all objective prerequisites for this.

\section{References}

1. A.V. Podzey, Mechanical Engineering, 180s. (1973)

2. M.A. Tamarkin, Science-intensive technologies in mechanical engineering, No. 3, (2018)

3. I.I. Shapovalov, Experience and economic aspects of industrial implementation of stabilizing treatment, pp. 96-101, (1985)

4. M.G. Babenko, S.V. Slesarev, Assessment of technological methods for stabilizing the accuracy of machine parts, pp. 75-77 (2015)

5. A.P. Babichev, I.A. Babichev, Fundamentals of vibration technology. -Rostov-n / D: DSTU, p. 620, (1999)

6. A.P. Babichev, The choice of optimal modes of vibration stabilizing treatment of body parts of a beam structure, Progressive finishing and hardening treatment: collection of articles, pp. 3-18, (1982)

7. M.A. Belozerov, V.A. Lebedev, Technical and natural sciences: innovations and prospects: materials of the international scientific-practical conference, Belgorod, January 30. 2020 - Belgorod: APNI, p. 96 (2020)

8. V.E. Antonyuk, Bulletin of the Belarusian State University of Transport: Science and Transport, № 1 (32), pp. 292-294, (2016)

9. V.A. Baskakov, Analysis of the propagation and dynamic impact of shock waves on a deformable solid: author. Dis. Dr. Phys. Math. Sciences: 01.02.04. - Cheboksary, p. 37, (1991)

10. I.A. Babichev, Model of shock impulse transmission in the ShSU system, Questions of vibration technology: interuniversity collection - Rostov-on / D: DSTU, pp. 9-21, (1991)

11. A.K. Efremov, Mechanical Engineering, p. 240, (1977)

12. M.A. Meyers, Metallurgy, p. 512, (1984)

13. F.M. Ferzullaev, Modern instrumental systems, information technologies and innovations: collection of scientific papers, pp. 200-204, (2019) 